\title{
QUANTITÉ ET PHRASÉOLOGIE: ASPECTS CULTURELS 1
}

\section{QUANTITY AND PHRASEOLOGY: CULTURAL ASPECTS}

\author{
Pierre-André Buvet ${ }^{2}$ \\ Université Paris 13 Sorbonne Paris Cité
}

Resumo: $\mathrm{O}$ artigo objetiva estabelecer a dimensão cultural da fraseologia em relação à expressão de quantidade. Quando é subjetiva, a codificação da quantidade é mais frequentemente baseada em unidades fraseológicas, na maioria das vezes, marcadas culturalmente. $\mathrm{O}$ artigo analisa sequências determinantes do valor quantificador para mostrar a dimensão cultural da fraseologia quando aplicada à determinação. O conceito de fraseologia é então usado para fundamentar os dados estudados.

Palavras-Chave: Fraseologia; Determinação; Cultura.

Abstract: The article aims to establish what is he cultural dimension of phraseology in relation to the expression of quantity. When it is subjective, the encoding of quantity is most often based on phraseological units, most often marked culturally. The article analyzes determinative sequences of quantitative value to show the cultural dimension of phraseology when applied to the determination. The phraseology matrix concept is then used to represent the data.

Keywords: Phraseology; Determination; Culture.

Article préparé en tant que produit du Projet Capes Cofecub 838/15.

2 Adresse e-mail de l'auteur: pierreandre.buvet@gmail.com. 
Résumé: L'article vise à établir quelle est la dimension culturelle de la phraséologie en rapport avec l'expression de la quantité. Lorsqu'elle est subjective, l'encodage de la quantité s'appuie le plus souvent sur des unités phraséologiques, le plus souvent marquées culturellement. L'article analyse des séquences déterminatives à valeur quantificatrice pour montrer la dimension culturelle de la phraséologie lorsqu'elle s'applique à la détermination. Le concept de moule phraséologique est ensuite utilisé pour représenter les données étudiées.

Mots-clés: Phraséologie; Détermination; Culture.

\section{INTRODUCTION}

Il est difficile de concevoir langue et culture indépendamment de leur implication réciproque comme l'attestent les propos suivants:

La langue est création. Elle n'est pas une sorte d'objet naturel, pour lequel on pourrait distinguer entre l'essence et le devenir, mais un objet culturel, plus particulièrement un processus de production culturelle, ce qui fait que le devenir fait partie de son essence. (COSERIU, 1980)

[On] n'apprend pas une langue pour en démonter les mécanismes et manipuler gratuitement des mots nouveaux, mais pour fonctionner dans la culture qui va avec cette langue, $[\ldots]$ celle-ci n'est pas une fin en soi, mais un moyen pour opérer culturellement, pour comprendre et produire du sens, avec les outils et dans l'univers de l'Autre. Donc que la culture, en tant qu'au-delà de la langue, est la fin recherchée. (GALISSON \& PUREN, 1999)

Les langues sont fondamentalement une partie de la culture, et on ne peut pas comprendre correctement les mots en les séparant des phénomènes culturels particuliers dont ils sont les symboles [...] Les mots sont fondamentalement des symboles pour les différents traits de la culture [...]. Il est évident que chaque langue contient un préfabriqué qui impose à ses locuteurs une certaine manière de regarder le monde, d'analyser l'expérience que nous avons du monde. (REY, 1980)

Les phraséologismes sont un sous-ensemble des faits de langue qui caractérisent une langue. Ils constituent une grande partie des discours, quels qu'ils soient, $c f$. SOUTET, MEJRI \& SFAR 2018. Ils sont très souvent révélateurs de l'intrication entre langue et culture que montrent les résonances entre les définitions suivantes de ces deux mots:

\section{Phraséologie}

'Ensemble des tournures typiques d'une langue, soit par leur fréquence, soit par leur caractère idiomatique' (Trésor de la Langue Française)

'Construction de phrase ou procédé d'expression propre à une langue, à une époque, à une discipline, à un milieu, à un auteur' (Larousse) 


\section{Culture}

‘Ensemble des phénomènes matériels et idéologiques qui caractérisent un groupe ethnique ou une nation, une civilisation, par opposition à un autre groupe ou à une autre nation' (Larousse)

Ensemble lié de manières de penser, de sentir et d'agir plus ou moins formalisées qui, étant apprises et partagées par une pluralité de personnes, servent, d'une manière à la fois objective et symbolique, à constituer ces personnes en une collectivité particulière et distincte (Wikipédia)

L'objectif de cet article est d'établir la dimension culturelle de la phraséologie du point de vue de l'expression de la quantité. Celle-ci peut être très factuelle, par exemple un carnet de dix dans Il a acheté un carnet de dix tickets mais aussi très subjective, par exemple un paquet de dans Aujourd'hui, il a donné un paquet de conseils à son entourage. Dans le second cas de figure, l'encodage de la quantité s'appuie le plus souvent sur des unités phraséologiques, souvent très marquées culturellement. Du point de vue quantitatif, le décodage du message intègre également son caractère subjectif par le biais de la langue et de la culture qui sont communes au locuteur et à son interlocuteur. Nous le montrons ici à propos de séquences déterminatives à valeur quantificatrice. Après avoir expliqué en quoi la phraséologie s'applique à la détermination, nous présentons les déterminants nominaux quantifieurs, puis nous précisons leurs particularités culturelles et nous discutons de la façon de les représenter dans une matrice phraséologique.

\section{PHRASÉOLOGIE ET DÉTERMINATION}

Après avoir rapidement précisé la notion de détermination dans nos travaux sur la question, nous expliquons comment la phraséologie s'applique à celle-ci.

Les grammairiens s'accordent sur le fait qu'en français la détermination relève de la syntaxe du substantif puisque tout nom employé en discours a besoin d'éléments qui l'actualisent: les déterminants. Il en est ainsi de ces, des, les et plusieurs qui s'analysent comme des déterminants du substantif livres dans les énoncés suivants: J'ai acheté ces livres; J'ai acheté des livres, J'ai acheté les livres; J'ai acheté plusieurs livres. La tradition limite la détermination aux éléments qui précèdent le nom, $c f$. RIGIEL, 1994. De ce point de vue, sont prototypiques de la détermination les articles défini, indéfini et partitif et se rajoutent aux articles les démonstratifs, les possessifs, les adjectifs indéfinis et les interrogatifs. Ces 
analyses sont partagées par toutes sortes de théories linguistiques qui font l'hypothèse que le syntagme nominal fonctionne selon le schéma suivant:

\section{Syntagme nominal $\Rightarrow$ déterminant + nom}

Nous défendons une autre hypothèse, $c f$. BUVET 2013 selon laquelle un groupe nominal est formé d'un substantif tête et de sa détermination. Le substantif-tête est le nom qui entretient des relations distributionnelles avec les autres constituants majeurs de la phrase, notamment le groupe verbal. Il s'ensuit que tout élément du groupe nominal qui n'est pas la tête nominale est constitutif de sa détermination en tant que déterminant. Autrement dit, la détermination d'un nom correspond à l'ensemble de ses déterminants. C'est donc un autre schéma qui est mis en avant ici:

\section{Groupe nominal => Prédéterminant + Nom + Modifieur}

Un prédéterminant est donc le déterminant ou la séquence de déterminants toujours présents à gauche des substantifs-têtes ${ }^{3}$, par exemple les ou trois des dans les exemples suivants: Les fils/Trois des fils/Les fils de la voisine/Trois des fils de la voisine/Les derniers fils de la voisine/ Les trois derniers fils de la voisine/Les derniers fils de la voisine du troisième étagel Les trois derniers fils de la voisine du troisième étage/Les derniers fils de l'ancienne voisine du troisième étage/Les trois derniers fils de l'ancienne voisine du troisième étage. Les autres déterminants constituent un modifieur, par exemple de, la, voisine, derniers, du, troisième, étage, ancienne dans ces exemples. L'ensemble des déterminants, qu'ils appartiennent à la catégorie du prédéterminant ou bien à celle du modifieur, sont constitutifs de la détermination du nom voisine.

Le groupe nominal est donc analysé ici comme une structure ternaire telle que l'élément occupant la position centrale (Nom) est obligatoire, l'élément occupant la position initiale (Prédéterminant) est obligatoire et l'élément occupant la position finale (Modifieur) a comme particularité de ne pas toujours saturer cette dernière position. Ce point est fondamental d'un point de vue phraséologique car il participe à la définition des moules phraséologiques en rapport avec la détermination.

L'absence de déterminant à gauche d'un nom est interprétée comme la marque de l'article zéro. Par conséquent l'article zéro est le prédéterminant dans Il a faim, cf. ANSCOMBRE 1986. 
La détermination simple est analysée comme un cas particulier de détermination complexe. C'est-à-dire une séquence déterminative discontinue prédéterminant_modifieur telle que le modifieur n'est pas réalisé. Il y a toutes sortes d'arguments pour cette analyse, faute de place, ils ne sont pas présentés ici, cf. BUVET Ibidem.

Les adjectifs épithètes antéposés sont les seuls modifieurs à gauche des substantifs-têtes, par exemple beaux dans les beaux gâteaux. Les autres sont à droite des substantifs-têtes. Rappelons qu'il s'agit de compléments du nom, par exemple de ma mère dans les gâteaux de ma mère, de relatives, par exemple que ma mère prépare dans les gâteaux que ma mère prépare, de participiale, par exemple, faits parmamère dans les gâteaux faits par ma mère, des infinitives, par exemple à se rouler par terre dans des gâteaux à se rouler par terre, et pour des substantifs spécifiques, des noms prédicatifs cf. GIRY-SCHNEIDER 1987, des complétives, par exemple qu'il aime les gâteaux de sa mère dans L'idée qu'il aime les gâteaux de sa mère m'a traversé l'esprit.

Le modifieur n'est pas un élément facultatif dans de nombreux cas de figure comme l'indiquent les exemples suivants: Il a eu la peur ( $E+$ de sa vie) ; Il avait une peur $(E+$ bleue $)$ de la maladie ; Il a une peur $\left({ }^{*} E+\right.$ panique $)$ des ascenseurs. Relativement au nom peur, les séquences discontinues la_de sa vie, une_bleue et une_panique forment un bloc déterminatif insécable. Les modifieurs et la structure ternaire qu'ils impliquent prouvent que la détermination est par nature complexe.

Le caractère complexe de la détermination se manifeste de toutes sortes de façons. En premier lieu, il y a la combinaison de prédéterminants, par exemple mes différents dans mes différents livres ou certains de mes dans certains de mes livres. On constate ici que des prédéterminants se combinent directement entre eux, tandis que d'autres imposent la présence du relateur de. Par ailleurs, les combinaisons de prédéterminants sont soumises à de fortes contraintes combinatoires. Ainsi, en français, il est impossible d'intervertir l'ordre des deux prédéterminants dans mes différents et certains de mes: *différents mes livres/*différents de mes livres/*mes certains livres/*mes de certains livres. En second lieu, il y a les associations prédéterminant-modifieur dont la liberté combinatoire est loin d'être aussi libre qu'il ne l'est dit dans les grammaires, $c f$. infra. En troisième lieu, il y a les séquences déterminatives, correspondant à : 1) des déterminants adverbiaux, par exemple beaucoup de dans beaucoup de livres; 2) une partie des déterminants adjectivaux, par exemple je ne sais quel dans je ne sais quel livre; 3 ) une partie des déterminants numéraux, par exemple 
trois mille trois cent trente-trois dans trois mille trois cent trente-trois livres; 4) les déterminants nominaux, par exemple une centaine de dans une centaine de livres. Cette complexité de la détermination explique sa dimension phraséologique. C'est particulièrement vrai pour les associations prédéterminant modifieur et les séquences déterminatives constituées d'éléments pluricatégoriels dont la combinatoire est fortement contrainte, c'est-à-dire qui ont à voir avec la congruence, la fixité et parfois l'opacité sémantique.

Ci-dessous une typologie de la détermination du français fondée sur des critères tant morphosyntaxiques que syntactico-sémantiques.

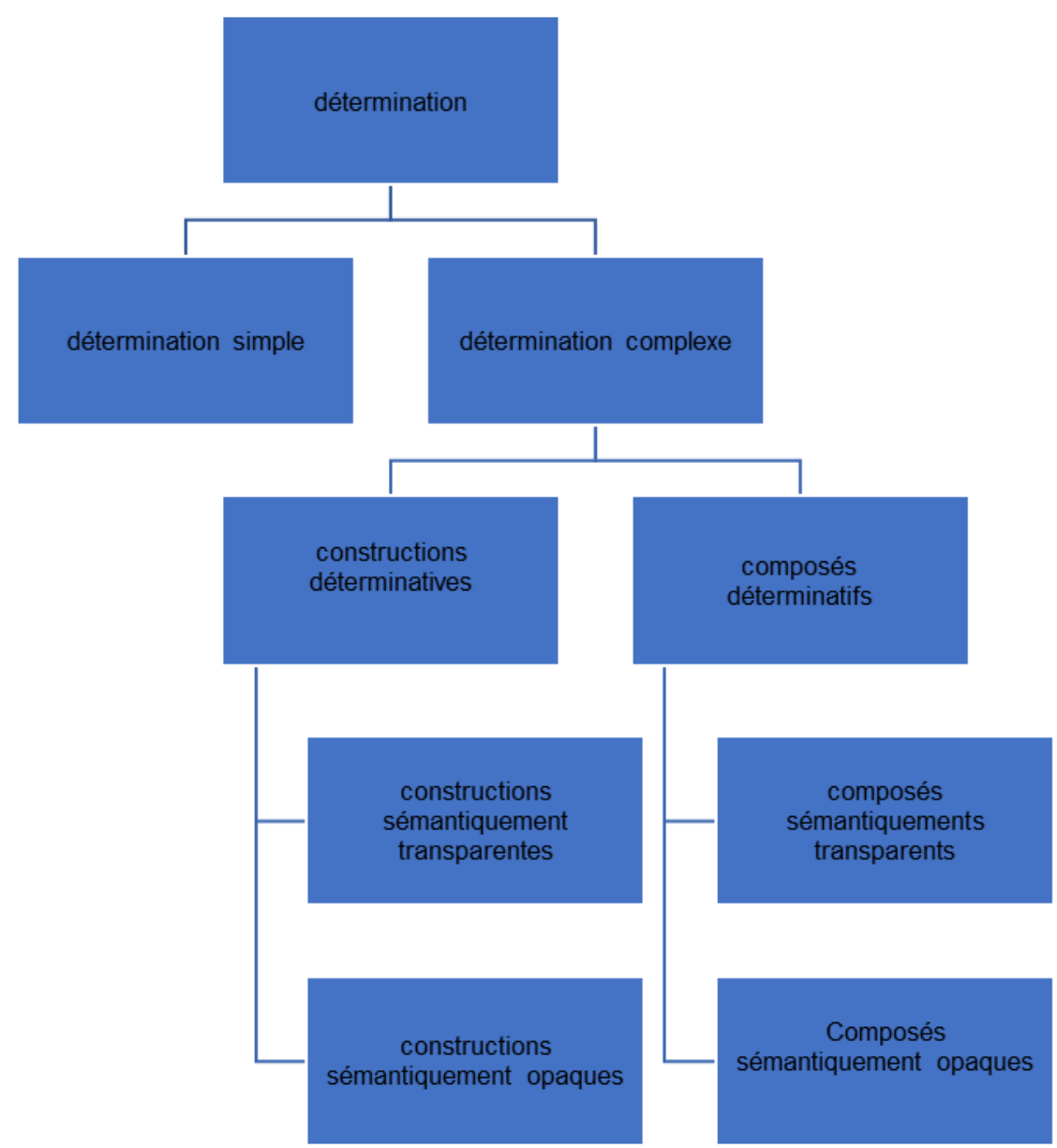

Fig. 1. Schéma d'une typologie.

Source: Schéma développé ad hoc par Pierre-André Buvet (2018)

La typologie témoigne de la nature fondamentalement complexe de la détermination et, par voie de conséquence, de son caractère phraséologique. Précisons ici ce qui distingue les constructions déterminatives des composés déterminatifs. Une construction déterminative est un assemblage d'unités déterminatives donnant lieu à une séquence déterminative constitutive d'une 
détermination. Ainsi, pris en compte globalement, un modifieur correspond à une unité déterminative, par exemple d'ange dans Il a une patience d'ange. L'association d'au moins deux unités déterminatives produit une séquence déterminative, qui est la condition minimale pour doter un nom d'une détermination. L'association du prédéterminant un et du modifieur d'ange forme la détermination du nom patience dans le dernier exemple.

Les constructions déterminatives sont distinguées selon leur degré de figement. Une première catégorie regroupe les constructions déterminatives qui sont sémantiquement transparentes. Elles comportent : 1) les combinaisons de deux prédéterminants, par exemple beaucoup de ces dans Il a rencontré beaucoup de ces personnes en allant en Irlande et Il a rencontré ces quelques personnes en visitant cet établissement ; 2) l'association d'un antédéterminant ${ }^{4}$ et d'un prédéterminant, par exemple la totalité de ces dans Il a rencontré la totalité de ces personnes et toutes ces dans Il a rencontré toutes ces personnes ; 3) l'association d'un prédéterminant et d'un modifieur par exemple, rouge dans Il a acheté une voiture rouge, de son frère dans Il a acheté la voiture de son frère, que j'ai mise en vente dans Il a acheté la voiture que j'ai mise en vente, que Léa vienne dans Il a parlé de la possibilité que Léa vienne, à vendre dans Il parle de la maison à vendre, vendue dans Il parle de la maison vendue et vendant des maisons dans Il parle de l'homme vendant des maisons. Une deuxième catégorie regroupe les constructions déterminatives qui sont sémantiquement opaques. On les observe uniquement lorsqu'il y a association entre un prédéterminant et un modifieur, par exemple les_coupés en brosse dans $\mathrm{Il}$ a les cheveux coupés en brosse, une_de guêpe dans elle a une taille de guêpe, un _en trompette dans Il a un nez en trompette et un_qui tire à hue et à dia dans On a affaire à un patronat qui tire à hue et à dia. Les séquences déterminatives de la catégorie des constructions déterminatives sémantiquement opaques ont souvent un caractère collocatif très marqué. C'est le cas notamment dans les quatre premiers exemples ci-dessus puisqu'elles s'appliquent à un et un seul nom, celui qu'elle détermine. Certains s'appliquent à plus d'un nom, c'est le cas de un _qui tire à hue et à dia: On a affaire à un (patronat + triumvirat + gouvernement + ministère...+$)$ qui tire à hue et à dia ${ }^{5}$.

La composition déterminative concerne des assemblages d'unités linguistiques qui ne sont pas toutes déterminatives mais qui donnent lieu à une séquence déterminative. Une première catégorie regroupe les composés

4 Un antédéterminant est une unité déterminative toujours antéposée au substantif-tête mais qui ne peut se combiner directement avec lui, cf. BUVET Ibidem.

5 Remarquons que les substantifs qui acceptent un_qui tire à hue et à dia comme détermination ont une certaine homogénéité sémantique. 
déterminatifs qui sont sémantiquement transparents. Il s'agit : 1) de l'ensemble des déterminants adverbiaux, c'est-à-dire des séquences déterminatives, formées d'un adverbe à valeur quantificatrice ou intensificatrice et de la préposition de, par exemple beaucoup de dans Il a lu beaucoup de livres et Il a eu beaucoup de chance; 2) de déterminants nominaux, c'est-à-dire des séquences formées a minima d'un prédéterminant, d'un nom déterminatif et de la préposition de, par exemple une bibliothèque de dans Il a lu une bibliothèque de livres, dont la valeur est la quantité précise, par exemple trois mètres de dans Il a acheté trois mètres de cordage, la quantité approximative, par exemple trois pelletée de dans Il a versé trois pelletées de goudron sur la chaussée ou l'aspect, par exemple un début de dans Il a un début de grippe, cf. infra ; 3) d'une grande partie des déterminants numéraux, par exemple trois cent trente-trois dans Il a lu cent trente-trois livres; une partie des déterminants adjectivaux, par exemple, je ne sais que dans Il l'a lu dans je ne sais quel livre.

Les composés déterminatifs qui sont sémantiquement opaques sont un sous-ensemble des déterminants nominaux. Ils servent également à spécifier la quantité, plus exactement la quantité approximative, par exemple une moisson de dans La France a ramené une moisson de récompenses, une montagne de dans Il a fait une montagne de fautes ou un nuage de dans Il s'est versé un nuage de lait dans son thé, l'aspect, par exemple une rafale de dans Il lui a posé une rafale de questions ou l'intensité, par exemple, une litanie de dans Une litanie de plaintes l'attendait dès son arrivée dans son bureau.

Qu'il s'agisse de constructions déterminatives ou bien de composés déterminatifs, la détermination complexe relève de la phraséologie dans la mesure où elle en a les particularités, $c f$. infra. Le caractère phraséologique de ces séquences déterminatives est d'autant plus marqué pour les constructions déterminatives et les composés déterminatifs qui sont sémantiquement opaques. En effet, leur degré de figement est plus important, du fait non seulement de leur opacité sémantique mais aussi de leur caractère collocatif, $c f$. BUVET 2014 et MEL'CUK 2003.

Désormais, seuls les déterminants nominaux à valeur quantificatrice sont pris en compte.

\section{DÉTERMINANTS NOMINAUX QUANTIFIEURS}

Selon Dubois et ali. 1994, "les déterminants sont les constituants du syntagme nominal qui dépendent du nom, tête ou constituant principal du syntagme nominal". Les substantifs en position initiale dans le groupe nominal 
peuvent fonctionner comme des déterminants; ce sont donc des noms déterminatifs. L'existence d'un nom déterminatif dans un groupe nominal est subordonnée à trois conditions: 1) il est précédé d'un prédéterminant; 2) il précède un autre nom; 3) les deux noms sont reliés par la proposition de. Un nom est déterminatif lorsqu'il est constitutif d'un déterminant nominal, c'est-àdire une séquence déterminative correspondant à la construction DET (MODIF) N (MODIF) $\mathbf{d e}^{6}$. Un déterminant nominal se combine soit directement avec le substantif qu'il détermine, par exemple un litre de relativement à vin dans Il a bu un litre de vin (type DET $\mathbf{N} \operatorname{de}_{1} \mathbf{N}$ ), soit par l'intermédiaire d'un autre prédéterminant, par exemple un litre de et ce relativement à vin dans Il a bu un litre de ce vin (type $\underline{\operatorname{DET} \mathbf{N} \operatorname{de}_{1}} \underline{\mathbf{D E T}_{2}} \mathbf{N}$ ).

Les déterminants nominaux sont donc directement associés avec la tête nominale. Ils donnent lieu alors à des groupes du type DET N de $\mathbf{N}$. Ils peuvent être combinés avec un autre type de prédéterminant, l'un des définis comme dans l'exemple ci-dessus ou un autre déterminant nominal comme dans Il a cassé environ trois dizaines de boites d'œufs. Ils donnent lieu alors à des GN du type DET $\mathbf{N}$ de 1 DET 2 . Dans le deuxième cas de figure, DET $\mathbf{N}$ de et DET ont le même statut. Ce sont des prédéterminants. Leur association est soumise à de fortes contraintes.

C'est l'environnement phrastique, dit également cotexte, qui établit si le nom en position initiale fonctionne comme un déterminant ou bien comme un substantif tête. C'est clairement le cas de kilo dans Il a acheté un kilo de farine. La paraphrase Il a acheté de la farine pour un kilo le confirme. Les noms déterminatifs ont rarement un statut unique, c'est-à-dire ils ne sont pas identifiables en tant que tels. Si cuiller est un nom qui n'est pas déterminatif dans J'ai acheté une cuiller en argent, ce n'est pas le cas dans J'ai versé une cuiller de farine. Leur configuration syntaxique est également insuffisante pour établir qu'il s'agit d'un nom déterminatif. Ainsi, la séquence un sac de farine a deux lectures différentes dans J'ai déchiré un sac de farine et J'ai versé un sac de farine. Dans le premier cas de figure, sac est la tête du GN car il est le seul des deux substantifs qui est compatible avec déchirer. Dans le second cas de figure, farine est la tête nominale car il est le seul des deux substantifs apparaissant dans le domaine d'arguments du prédicat verser et, de ce fait, sac correspond à un nom déterminatif. La possibilité d'une double lecture des noms déterminatifs est très générale. Les noms uniquement déterminatifs sont peu nombreux. C'est le cas

6 DET est le code pour déterminant, MODIF pour modifieur, $\mathrm{N}$ pour nom. DET ici correspondant à la détermination du déterminant nominal, $c f$. BUVET Ibidem. 
de kyrielle dans J'ai lu une kyrielle d'articles sur ce sujet ou de floppée dans J'ai vu une floppée de documentaires qui en parlent.

Les règles d'accord contreviennent souvent à l'analyse syntacticosémantique des noms déterminatifs. Par exemple dans Un gros paquet de clous (a + ${ }^{*}$ ont) été utilisé pour fixer cette planche, l'accord du verbe se fait avec le nom déterminatif paquet (qui est singulier) au lieu de la tête nominale clous (qui est pluriel). De même, dans Trois litres d'eau (se sont $+{ }^{*}$ s'est) déjà évaporés, l'accord du verbe se fait avec le nom déterminatif litres (qui est pluriel) au lieu de la tête nominale eau (un nom massif donc nécessairement au singulier ${ }^{7}$ ). Ce n'est pas le cas dans cet autre exemple: Un grand nombre de supporters $(? a+o n t)$ violemment sifflé l'arbitre. L'accord du verbe se fait ici avec la tête nominale supporters (qui est pluriel) au lieu de la tête nominale grand nombre (qui est au singulier). Par contre, c'est le cas dans cette variante de l'exemple précédent : Le grand nombre de supporters a violemment sifflé l'arbitre. L'accord se fait ici avec le nom déterminatif, parce que l'article défini est la composante majeure de la séquence déterminative le grand nombre de. D'une façon générale, lorsqu'il y a combinaison de prédéterminants, c'est celui qui est antéposé qui impose ses propriétés syntaxiques et sémantiques à l'ensemble de la séquence, $c f$. BUVET Ibidem.

Ci-dessous une typologie morphosémantique des noms déterminatifs:

- nom de nombre; par exemple centaine dans Il achète une centaine de bons du trésor

- unité de mesure de longueur; par exemple mètre dans Il lui a achète un mètre de ruban

- unité de mesure de surface; par exemple mètre carré dans Il emporte un mètres carré de moquette

- unité de mesure de masse; par exemple kilogramme dans Il a donné un kilogramme de viande à son chien

- unité de mesure de volume Il a bu un litre de vin

- monnaie Il a volé cinq francs de bonbons

- contenant Il a contrôlé l'identité d'un car entier de réfugiés

- suffixé en -ée Il a livré plusieurs charretées de foin à Max

- surface Il commande un plateau d'huîtres

- support Il a traité une clef usb de données

- collectif Il commande trois régiments de zouaves

Il existe quelques noms massifs nécessairement au pluriel, par exemple épinards. Ils sont très rares. 
- forme Il a mangé trois boules de glace

- partie Il a mangé trois morceaux de gâteaux

- fraction Il m'a vendu trois dixièmes de ce gâteau

- nom de temps $I l$ a trois heures de retard

- substantif déverbal Il remarque une accumulation de fautes

- déterminant figé Il a fait une montagne de fautes d'orthographe

Des propriétés des déterminants nominaux sont rapidement présentés cidessous. Elles corroborent leur dimension phraséologique.

La première propriété est la possibilité de réduire une séquence DETa Na de (DETb) Nb, telle que DETa Na de est un déterminant nominal, à une séquence DETa Na lorsqu'il y a des contraintes de sélection entre un prédicat et son argument. C'est le cas de trois paquets de cigarettes dans Il a fumé trois paquets de cigarettes; on peut réduire le groupe nominal à trois paquets: Il a fumé à trois paquets. La spécificité de la relation distributionnelle entre fumer et cigarette dans Il a fumé trois paquets de cigarettes explique l'effacement du substantif-tête. La substitution de fumer par parler, un verbe compatible avec toutes sortes de compléments, le confirme car elle va de pair avec l'impossibilité de réduire la phrase Il a parlé de trois paquets de cigarettes à Il a parlé de trois paquets $^{8}$. On peut également effacer la tête nominale lorsque sa relation avec le nom déterminatif est de nature collocative. C'est le cas, par exemple de la séquence un quatuor de violonistes dans Il parle d'un quatuor de violonistes; elle est réductible à la séquence un quatuor: Il parle d'un quatuor'. Le substantif quatuor étant un nom collectif d'humains, plus précisément, de musiciens ${ }^{10}$. Il s'ensuit que la réduction de Il parle d'un quatuor de violonistes est consécutive à la spécificité de la seule relation entre quatuor et violoniste. Par contre, dans Il parle d'un quatuor de politiciens, l'effacement du second substantif est interdit car politicien n'apparaît pas parmi les noms de musiciens.

La seconde propriété est la possibilité de répéter un nom déterminatif précédé de des. L'article indéfini pluriel en tant que déterminant interne des déterminants nominaux conduit souvent à les interpréter d'une façon quasi-

8 L'énoncé Il a parlé de trois paquets n'est pas incorrect mais il n'est pas nécessairement l'équivalent de Il a parlé de trois paquets de cigarettes. Les facteurs pragmatiques ne sont pas pris en compte ici.

9 La présence de parler dans Il parle d'un quatuor de violonistes permet d'observer que l'effacement de la tête nominale n'est pas imputable aux restrictions de sélection entre le verbe et son complément.

10 Ce dernier point est attesté par l'effet de redondance que produit l'adjonction de musiciens à quatuor: Il parle d'un quatuor de musiciens. 
identique indépendamment de leur nature: Il a acheté des (mètres de tissu + litres d'huile+ sacs de friandises + rayons de livres). Les séquences des mètres de, des kilos de, des sacs de et des rayons de sont sémantiquement équivalentes dans le premier cas de figure. Elles signifient approximativement 'une grande quantité de', car des devant le nom déterminatif tend à annihiler la valeur de ce dernier tout en uniformisant celle du déterminant nominal. Cette particularité de des n'implique pas pour autant une altération des contraintes distributionnelles entre le nom déterminatif et la tête nominale: Il a acheté des ((mètres $+{ }^{*}$ litres + sacs + ? rayons $)$ tissu $+\left({ }^{*}\right.$ mètres + litres $+{ }^{*}$ sacs $+{ }^{*}$ rayons $)$ d'huile $+\left({ }^{*}\right.$ mètres $+{ }^{*}$ litres + sacs + ?rayons $)$ de friandises $)+\left({ }^{*}\right.$ mètres $+{ }^{*}$ litres + sacs + rayons $)$ de livres $\left.)\right)$. L'amoindrissement de la valeur des noms déterminatifs est renforcé si ces derniers sont redoublés par l'intermédiaire d'une coordination en et: Il a acheté (des mètres et des mètres de tissu + des litres et des litres d'huile + des sacs et des sacs de friandises + des rayons et des rayons de livres. L'homogénéisation sémantique des noms déterminatifs précédés de des est donc d'autant plus grande qu'il apparaît deux fois dans la séquence déterminative.

La troisième propriété est la possibilité, dite restructuration, de substituer le déterminant nominal par une construction prépositionnelle, les deux constructions ayant la même valeur: Il a mangé des pleins bocaux de ces cerises/Il a mangé de ces cerises par pleins bocaux, cf. GUILLET et LECLERE 1981. Outre les restructurations en par, il y a des restructurations en $\grave{a}$, par exemple la paire Il a reçu une foison de cadeaux/Il a reçu des cadeaux à foison, des restructurations en en, par exemple la paire La radio a annoncé une cascade de décès/La radio a annoncé des décès en cascade, des restructurations en de, par exemple la paire Il a une heure de retard/Il a un retard d'une heure. Une telle restructuration dépend du nom déterminatif. Si la restructuration en par est relativement productive, c'est moins le cas pour les restructurations en à et en en. La restructuration en de est limitée aux noms déterminatifs de la classe unités de temps. Ces restructurations sont soumises à conditions en rapport avec la détermination interne, les particularités syntaxiques de la tête nominale et la nature des verbes dont elle dépend.

\section{DIMENSION CULTURELLE}

Ensemble des aspects intellectuels, esthétiques et éthiques d'un groupe humain bien identifié, d'abord appliqué à une nation ou à un ensemble systématique des formes acquises de comportement dans toute société humaine, incluant outre la symbolique sociale consciente, les croyances et les valeurs assumées, les normes sociales, mais aussi les habitudes matérielles de toute nature, les comportements 
les plus variés, pour peu qu'ils soient socialisés et portent la marque d'une symbolique. Selon une telle définition, une culture englobe tout le système de valeurs dominantes et des modes de comportement d'une société, et par exemple les techniques, les habitudes corporelles etc. (REY 1987).

Si l'on admet que les phraséologismes témoignent de la dimension culturelle de la langue française, cf. REY \& CHANTREAU, 1989, la question centrale est d'intégrer le facteur culture, en tant que système évolutif de règles et de conventions, dans le formalisme descriptif et explicatif de la phraséologie. Ce formalisme s'appuie ici sur le concept de moule phraséologique. Il s'agit donc d'établir comment les données culturelles, combinées aux données linguistiques, sont décrites dans un moule phraséologique. De ce point de vue, les déterminants nominaux quantifieurs s'avèrent être pertinents pour tenter de répondre à cette question dans la mesure où leur analyse est relativement simple.

Un moule phraséologique est défini comme une structure constituée d'éléments qui sont invariants et d'autres qui ne le sont pas. Cette structure a pour fonction d'expliquer le mode de formation des phraséologismes. Les éléments invariants sont décrits avec des métarègles, les éléments faisant l'objet de variation sont décrits avec des règles. Les métarègles et les règles ont pour rôle d'expliquer le mode de fonctionnement du moule phraséologique, $c f$. BUVET 2018.

Les métarègles font état des principes généraux qui sous-tendent la formation des phraséologismes. Elles sont de nature sémantique, d'une part, morphosyntaxique, d'autre part.

Deux métarègles s'appliquent au moule phraséologique des déterminants nominaux quantifieurs.

\section{Métarègle1 $:$ DNOM = ACTUALISATEUR}

La métarègle 1 est de nature sémantique. De ce point de vue, le traitement des moules phraséologiques s'inscrit dans le cadre de la théorie des trois fonctions primaires selon laquelle les unités linguistiques s'expliquent en termes de fonction prédicative, de fonction argumentale ou de fonction modalisatrice, cf. BUVET 2016, MEJRI 2016, MEJRI 2017 et MEJRI 2018. Fondamentalement la détermination relève de la fonction modalisatrice. C'est pourquoi les déterminants nominaux sont définis ici comme des actualisateurs. 


\section{Métarègle 2 : DET (MODIF) N (MODIF) de}

La métarègle 2 est de nature morphosyntaxique. Elle définit la construction générale des déterminants nominaux. Ici elle indique la nature des éléments de la construction, leur position et leur caractère obligatoire ou facultatif. Un déterminant nominal comporte nécessairement un nom déterminatif précédé d'un prédéterminant et suivi de la préposition. Il y a des noms déterminatifs qui excluent tout modifieur, comme tas dans Ce livre propose un tas de solutions, des noms déterminatifs qui imposent un modifieur antéposé ou postposé comme nombre dans Ce livre répond à un grand nombre de nos questions et Ce livre répond à un nombre incroyable de nos questions et des noms déterminatifs qui n'excluent pas et n'imposent pas un modifieur antéposé ou postposé comme foule dans Une foule de supporteurs attendait les joueurs/Une incommensurable foule de supporteurs attendait les joueurs/ Une foule considérable de supporteurs attendait les joueurs.

Le moule phraséologique prend en compte les contraintes lexicales, les contraintes syntaxiques et les contraintes sémantiques des déterminants nominaux. Ces contraintes sont formulées en termes de règles et de sous-règles.

Une règle est définie en termes de condition et de réalisation. Les règles 1 à 3 spécifient la valeur générale du déterminant nominal en fonction du nom que le prédéterminant précède.

\section{$\underline{\text { Règle 1 }: ~} \mathbf{N}=$ Nargumental $=>$ VALEUR $($ DNOM $)=$ QUANTITE}

Dans la règle 1 , la condition concerne la nature du nom, il s'agit d'un nom argumental, cf. BUVET 2013. La réalisation se manifeste sous la forme de valeur du déterminant nominal, il s'agit d'une quantité. Cette règle explique, par exemple, la valeur quantificatrice du déterminant nominal trois bocks de dans Il a bu trois bocks de bière. Des sous-règles de la règle 1 expliquent la combinatoire entre le déterminant nominal et le nom qu'il précède. Par exemple, quels types de noms sont compatibles avec les noms de contenant. Par exemple, des noms de moyen de transport du type car se combinent plutôt avec des noms d'êtres humains alors que ceux du type bétaillère se combinent plutôt avec des noms d'animaux. Elles détaillent également, les différentes valeurs quantificatrices possibles: quantité précise, quantité approximative importante, quantité approximative faible, $c f$. supra. 


\section{$\underline{\text { Règle 2 }}: \mathbf{N}=$ Nprédicatif + massif $\Rightarrow$ VALEUR $($ DNOM $)=$ INTENSITE}

Pour ce qui est de la règle 2 , la condition concerne la nature du nom, il s'agit d'un nom prédicatif, $c f$. BUVET Ibidem, doté du trait massif; $c f$ KLEIBER 1990. La réalisation se manifeste sous la forme de valeur du déterminant nominal, il s'agit d'une intensité. L'intensité est considérée comme un cas particulier de quantité, $c f$. BLANCO 2009. C'est le cas du déterminant nominal une bonne dose de dans Il a fait preuve d'une bonne dose de patience. Les sous-règles associées à cette règle permettent de distinguer l'intensité forte de l'intensité faible.

\section{$\underline{\text { Règle 3 }}: \mathrm{N}=$ Nprédicatif + comptable $=>$ VALEUR $($ DNOM $)=$ ASPECT}

La règle 3 caractérise les déterminants nominaux qui se combinent avec les noms déterminés correspondant à des noms prédicatifs dotés du trait comptable, $c f$. KLEIBER Ibidem. Elle spécifie que leur valeur est de nature aspectuelle. L'aspect est une valeur en rapport avec la temporalité, $c f$. GUILLAUME 1929. Qui concerne également la quantité dans la mesure où l'aspect exprime une unicité temporelle, une itération temporelle ou une fréquence temporelle. C'est le cas, par exemple, du déterminant nominal une floppée de dans Il lui a donné une floppée de conseils. Les sous-règles en rapport avec la règle 3 précisent les différentes valeurs de l'aspect: le semelfactif, l'itératif ou le fréquentatif.

\section{Règle 4 : N= Nprédicatif + comptable + massif =>VALEUR $($ DNOM $)=$ ASPECT}

Les noms déterminés correspondant à des noms prédicatifs compatibles avec les traits comptable ou massif sont concernés par la règle 4 . Les sous-règles en rapport avec cette règle précisent la valeur de l'aspect: 1) l'inchoatif, par exemple un commencement de dans Il m'a donné un commencement de réponse ou un début de dans Il a eu un début de grippe 2) le terminatif, par exemple une fin de dans L'équipe de France a fait une remarquable fin de parcours; 3) le continuatif; par exemple une longue période de dans Il a pris une longue période de repos. 


\section{$\underline{\text { Règle } 5:} \mathbf{N}=$ Ndet $\rightarrow$ Co(n)texte}

La règle 5 stipule la nécessité d'un contexte/cotexte pour établir le caractère déterminatif du nom, cf. supra. Par exemple, le substantif car, en tant que substantif constitutif d'une séquence DET $\mathbf{N}$ de, n'est pas déterminatif dans J'ai doublé un car de touristes mais il l'est dans Le douanier a contrôlé tout un car de touristes.

\section{$\underline{\text { Règle 6 }}$ : Ndet $\rightarrow$ *Co(n)texte}

La règle 6 est l'opposée de la règle 5. Elle précise qu'aucun contexte/cotexte n'est pas nécessaire pour établir le caractère déterminatif du substantif constitutif d'un déterminant nominal. Par exemple, le substantif tripotée, en tant que substantif constitutif d'une séquence DET $\mathbf{N}$ de, est déterminatif aussi bien dans J'ai doublé une tripotée de touristes que dans Le douanier a contrôlé une tripotée de touristes.

Faute de place, l'ensemble des règles n'est pas mentionné ici. Il est question désormais des déterminants nominaux figés pour discuter des pistes de recherche envisagées pour formaliser la dimension culturelle dans les moules phraséologiques. Le traitement de cet aspect des langues dans la phraséologie, et plus particulièrement du point de vue des déterminants nominaux quantifieurs, s'articule autour de trois axes: 1) leur caractère idiomatique; 2) l'opposition langue écrite vs langue orale; 3) la situation d'énonciation.

Pour ce qui est du premier axe, rappelons que « Le terme d'idiome désigne fort justement la langue comme reflétant les traits propres d'une communauté » SAUSSURE 1916. De ce point de vue, le concept de collocatif est fondamental, car il caractérise les expressions idiomatiques, $c f$. MEJRI 2008. Il en est ainsi, par exemple, de la séquence un tonnerre de dans Il a été accueilli avec un tonnerre d'applaudissement, de la séquence une montagne de dans Il a fait une montagne de fautes et de la séquence un train de dans Le gouvernement a pris un train de mesures pour y remédier. Ces trois séquences s'appliquent respectivement et exclusivement aux noms déterminés applaudissements, fautes et mesures. Il s'ensuit le caractère hautement idiomatique des déterminants nominaux composés. La majorité d'entre eux relève de ce cas de figure. Un nombre plus limité s'avère peu ou pas collocatif. Par exemple, la séquence une litanie de dans Il évoquait une litanie de (questions + griefs + bonnes intentions $+\ldots$ ) ou la séquence 
des pelletées de dans On s'attendait à des pelletées de (problèmes + bons sentiments + musiciens $+\ldots)$. Les déterminants nominaux une litanie de et une pelletée de ont comme particularité d'être exclusivement déterminatifs, cf. supra.

En ce qui concerne le deuxième axe, les déterminants nominaux se répartissent très souvent selon qu'ils s'emploient plutôt à l'écrit ou plutôt à l'oral. Les premiers relèvent plutôt de la langue soutenue, par exemple la séquence son cortège de dans J'ai appris le latin avec son cortège de déclinaisons., la séquence une palanquée de dans Son article comportait une palanquée de références scientifiques et la séquence un tombereau de dans Il déversa sur eux un tombereau d'injures. Les seconds relèvent plutôt de la langue courante voire familière, par exemple la séquence un kil de dans Il s'est sifflé un kil de rouge, la séquence un iota de dans Il n'y pas un iota de différence entre nous et la séquence un brin de dans Il a fait un brin de causette avec sa voisine.

Les déterminants nominaux relevant de la langue orale nécessitent de tenir compte de la situation d'énonciation, c'est-à-dire les conditions pragmatiques de l'énoncé: le locuteur, l'interlocuteur, le contexte spatiotemporel, $c f$. NOLKE 1994. Il s'agit du troisième axe. Ainsi, il existe une série de déterminants nominaux prototypiques des discours oraux en rapport avec l'alimentation et la boisson et mettant en jeu des situations d'énonciation particulières (un hôte et son invité, un prescripteur et son auditoire): la séquence un doigt de dans Je vous sers un doigt de whisky, la séquence un fond de dans Mettre un fond de moutarde, la séquence trois gouttes de dans Verser trois gouttes d'essence de lavande, la séquence une pincée de dans Mettre une pincée de sel, la séquence un filet de dans Ajouter un filet de citron, la séquence une fine couche de dans Poser une fine couche de sucre glace. Signalons également la séquence une lampée de dans Il s'est enfilé une lampée de vin.

Intégrer la dimension culturelle dans un moule phraséologique consisterait à préciser en quoi elle interfère avec les conditions d'emploi des phraséologismes. Il s'agirait alors de formuler les conditions de nature culturelle dans les règles afin de spécifier en quoi elles sont nécessaires à la réalisation des phraséologismes ${ }^{11}$. Pour poursuivre ce travail sur l'expression de la quantité en rapport avec les phraséologismes et leur dimension culturelle, d'autres sortes de séquences déterminatives figées sont en cours d'étude. Par exemple, la séquence discontinue de l'_comme quatre dans Il a de l'esprit comme quatre, la séquence discontinue des_à foison dans Il a des idées à foison ou des_comme s'il en pleuvait dans Il a reçu des claques comme s'il en pleuvait.

11 Ce serait le cas pour expliquer les emplois des pragmatèmes, cf. BLANCO et MEJRI 2018. 


\section{PERSPECTIVES}

Tout discours serait fondé sur une opposition inhérente aux êtres humains: l'être subjectif vs l'être social, GRELLIER 2005. Ces deux aspects sont si indissociables qu'il est difficile d'établir quel est l'aspect le plus prégnant chez un individu. Cette opposition se manifeste de deux façons sur le plan linguistique: d'une part, des énoncés exprimant un ressenti psychologique ou physiologique, un sentiment ou une opinion personnelle sur un élément du monde extérieur, cf. BUVET 2014; d'autre part, des énoncés stéréotypiques, à $l^{\prime}$ instar des formes sentencieuses, $c f$. ANSCOMBRE 2008.

Dans les deux cas de figure, l'emploi des phraséologismes est très fréquent. Par exemple, l'être subjectif transparait lorsque le locuteur emploie le phraséologisme une faim de loup dans J'ai une faim de loup et l'être social est sollicité lorsque le locuteur mobilise les phraséologismes Revenons à nos moutons et toute la nuit n'y suffirait pas dans Toutefois, revenons à nos moutons car s'il fallait faire l'anthologie de la mauvaise gestion des programmes fédéraux, toute la nuit n'y suffirait pas. Les phraséologismes présents dans les énoncés centrés sur l'être subjectif et les énoncés émanant de l'être social tendent à prouver que ces derniers impriment leur marque sur les premiers. Si l'on admet que tout phraséologisme est un objet culturel, y recourir dans un énoncé éminemment subjectif, implique alors que celui qui est également formulé par l'être social, car le locuteur mobilise dans la culture de sa langue une expression qui sert à la manifestation de sa subjectivité.

Il s'agit d'une hypothèse de travail. Elle reste à valider dans les études à venir sur le concept de moule phraséologique du point de vue culturel.

\section{RÉFÉRENCES}

ANSCOMBRE, J.-C., L'article zéro en français: un imparfait du substantif?, Langue française, Paris, 72, p. 4-41, 1986.

ANSCOMBRE, J.-C., Les formes sentencieuses: peut-on traduire la sagesse populaire, META, 53-2, p. 62-75, 2008.

BLANCO, X., Valeurs grammaticales et structures prédicat-argument, Langages, Paris, 176, p. 50-62, 2009.

BLANCO, X. et MEJRI, S. Les pragmatèmes. Paris: Classique Garnier, 2018.

BUVET, P.-A. Collocation et expression de l'intensité. In: SOUTET, Olivier; MEJRI, Salah; SFAR, Inès (dir.). La phraséologie: théories et applications. Paris: Champion, 2018. p. $121-136$.

BUVET, P.A. La dimension lexicale de la détermination en français. Paris: Champion, 2013. 
BUVET, P.-A. Des unités lexicales aux unités discursives: la catégorisation sémanticoénonciative des prédicats. In: CAMPENHOUDT, Marc Van. L'unité en sciences $d u$ langage: Neuvièmes journées scientifiques du réseau thématique Lexicologie, Terminologie, Traduction. Paris: Archives Contemporaines, 2014. p. 154-178.

BUVET, P.-A. La fonction argumentale au regard des noms d'artefact. Actes du colloque CMLF 2016. Paris, 2016.

COSERIU, E. Vom Primat der Geschichte, Sprachwissenschat. München, 5, p. 125-145, 1980.

DUBOIS, J., M. GIACOMO, L. GUESPIN, C. MARCELLESI, J.-B. MARCELLESI. Dictionnaire de linguistique et des sciences du langage. Paris: Larousse, 1994.

GALISSON, R. et PUREN, C. La Formation en question. Collection Didactique des langues étrangères, Paris: CLE International, 1999.

GIRY-SCHNEIDER, J. Les Prédicats nominaux en français. Les Phrases simples à verbe support. Geneva: Droz, 1987.

GUILLAUME, G. Temps et verbe. Paris: Champion, 1929.

GUILLET, A et LECLERE, C., Restructuration du groupe nominal, Langages, 63, p. 99$125,1981$.

KLEIBER, G. L'article LE générique. La généricité sur le mode massif. Genève: Droz, 1990.

MEJRI, S., Constructions à verbes supports, collocations et locutions verbales, Rencontres méditerranéennes, 1, CERES, Tunis, p. 331-347, 2008.

MEJRI, S., Le prédicat et les trois fonctions primaires. In: COSTA, Daniela de Douza Silva; BENÇAL, Dayme Rosane. Nos caminhos do léxico. Campo Grande: Editora UFMGS, 2016, p. 313-337.

MEJRI, S. Les trois fonctions primaires. Une approche systématique. De la congruence et de la fixité dans le langage. In: CARVALHO, C., PLANELLES IVÁÑEZ, M., SANDAKOVA, E. De la langue à l'expression: le parcours de l'expérience discursive. Alicante: Universidad de Alicante, 2017, p. 123-144.

MEJRI, S. L'unité lexicale au carrefour du sens. La troisième articulation du langage. In: BLANCO, Xavier; SFAR, Inès. Lexicologie(s): approches croisées en sémantique lexicale. Suisse: Peter Lang, 2018. p. 18-32.

MEL'CUK, I. Collocations dans le dictionnaire. In: SZENDE, Tamás. Les écarts culturels dans les Dictionnaires bilingues. Paris: Champion, 2003.

NOLKE, H. Linguistique modulaire: de la forme au sens. Paris: Peeters, 1994.

REY, A. et CHANTREAU, S. Le dictionnaire des expressions figées. Paris: Editions Le Robert. 1989.

REY, A. Dictionnaire alphabétique et analogique de la langue française. Paris: Editions Le Robert. 1987.

SAUSSURE, F. de. Cours de linguistique générale. Paris: Payot, 1916.

Nota do editor:

Artigo submetido para avaliação em: 06 de dezembro de 2018.

Aprovado em sistema duplo cego em: 18 de dezembro de 2018. 